\title{
Effect of a new surface-grafting method for nano-hydroxyapatite on the dispersion and the mechanical enhancement for poly(lactide-co-glycolide)
}

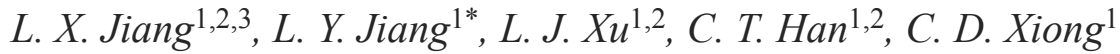 \\ ${ }^{1}$ Chengdu Institute of Organic Chemistry, Chinese Academy of Sciences, 610041 Chengdu, PR China \\ ${ }^{2}$ Graduated School of Chinese Academy of Sciences, 100039 Beijing, PR China \\ ${ }^{3}$ Shenzhen Institute of Advanced Technology, Chinese Academy of Sciences, 518067 Shenzhen, PR China
}

Received 19 August 2013; accepted in revised form 14 October 2013

\begin{abstract}
In this paper, a new surface-grafting D, L-lactide (DLLA) for nano-hydroxyapatite (n-HA) with the assist of citric acid was designed. The dispersion of new surface modified n-HA was characterized by Fourier transformation infrared (FTIR) spectroscopy, thermal gravimetric analysis (TGA), X-ray powder diffraction (XRD), transmission electron microscopy (TEM) and dispersion test, and the mechanical enhancement effect for poly(lactide-co-glycolide) (PLGA) was evaluated by scanning electron microscopy (SEM), differential scanning calorimeter measurements (DSC) and electromechanical universal tester. The results showed that citric acid played a critical role in surface-grafting, which could greatly increase grafting amount and improve dispersion of n-HA, so that it resulted in better interfacial adhesion throughout PLGA matrix, higher crystallinity and better mechanical enhancement for PLGA than the surface-grafting method for n-HA without citric acid, whose bending strength and tensile strength were both over $20 \%$ higher than those of pure PLGA when $3 \mathrm{wt} \% \mathrm{n}$-HA was added, and it still enhanced 8 and $6 \%$ higher than those of pure PLGA even the introduction of $15 \mathrm{wt} \%$ n-HA, respectively. The above results suggested that the surface-grafting for n-HA with the aid of citric acid was an ideal novel surface modification method, which could greatly improve the dispersion of n-HA and exhibit excellent mechanical enhancement effect for PLGA, suggesting it has a great potential in the bone fracture internal fixation application in future.
\end{abstract}

Keywords: polymer composites, nanocomposites, nano-hydoxyapatite, surface modification, mechanical properties

\section{Introduction}

Poly (lactic acid-co-glycolic acid) (PLGA) has been widely studied as bone fracture internal fixation materials [1]. However, the relatively poor mechanical property is insufficient to apply in dense bone, and the lack of bioactivity is unfavorable to bone fracture healing quickly $[2,3]$. While nano-hydroxyapatite (n-HA) is similar to the inorganic component of natural bone, which not only has good biocompatibility and osteo-conductivity, but also has good mechanical enhancement effect for polymer as nanoparticles $[4,5]$. Therefore, extensive efforts have been devoted to the research of introducing n-HA to
PLGA, which is expected to enhance its mechanical property and improve its bioactivity [6-8].

However, the incorporation of unmodified n-HA would decrease mechanical property of PLGA, due to the agglomeration of the n-HA in the PLGA matrix and the lack of adhesion between the n-HA particles and the PLGA matrix [9], which might limit the application of PLGA as bone fracture internal fixation materials. So surface-modification for n-HA is a vey key technique. Consequently, various surface modifications for n-HA have been developed, such as silane coupling agents, zirconyl salt, polyacids, isocyanate, dodecyl alcohol and lac-

\footnotetext{
${ }^{*}$ Corresponding author, e-mail: jlytxg@163.com

(c) BME-PT
} 
tide (LA) [10-14]. Among the above surface modification for n-HA, grafting ring-opening polymerization of LA onto the surface of n-HA particles is a very effective way to improve the interfacial affinity of n-HA to the PLGA matrix. However, when n-HA is directly treated by LA, the grafting amount is still low because of the poor reactivity of the hydroxyl groups on the surface of n-HA and the bad dispersion of n-HA in hydrophobic solvent. Accordingly, the mechanical enhancement effect for PLGA isn't ideal when higher n-HA content was added into PLGA [15]. However, generally speaking, the higher addition content of n-HA, the better osteological bioactivity of PLGA [16]. Therefore, new surface-grafting methods for n-HA should be explored so as to have both better mechanical enhancement effect and higher n-HA addition content for PLGA. In our previous study, we have investigated some surface-grafting methods for n-HA. For example, we found that the surface modification method of combining stearic acid with surface-grafting L-lactide was an effective modification method for n-HA, where stearic acid could improve the dispersion of n-HA during surface-grafting L-lactide, so the addition of $3 \mathrm{wt} \% \mathrm{n}$-HA into PLGA had the best mechanical enhancement for PLGA, while the addition of $15 \mathrm{wt} \% \mathrm{n}-\mathrm{HA}$ decreased markedly its bending strength $[17,18]$. Subsequently, we designed another surface-grafting method of combining silane coupling reagent (KH550) with surface-grafting L-lactide (L-LA), where KH550 not only could improve the hydrophobicity of n-HA acting as coupling agent but also could provide amino group to graft-polymerize with L-lactide onto the n-HA surface, so the mechanical enhancement effect was far more excellent than corresponding unmodified n-HA, however, the maximum addition content of n-HA into PLGA was only $10 \mathrm{wt} \%$ [19].

Based on the above results, to further improve the dispersion of n-HA and the mechanical enhancement for PLGA by the addition of higher n-HA content, a new surface-grafting method with the assistance of citric acid was reported in this paper, where molecular structure of citric acid has three carboxyl groups and one hydroxyl group, and the steric hindrance of citric acid is conducive to improve the dispersion of n-HA, so it is expected to improve grafting amount for $\mathrm{n}-\mathrm{HA}$ by forming a Ca carboxylate bond with citric acid, and D, L-lactide could be ring-opening graft-polymerized onto the n-HA sur- face by the hydroxyl group of citric acid, based on the literature [20]. Moreover, the new surface-grafted n-HA was introduced into PLGA matrix with different contents of 3, 8, 15 and $25 \mathrm{wt} \%$ to investigate the mechanical enhancement effect for PLGA, comparing with the surface-grafted n-HA without citric acid. The main aim of the study is to provide an ideal novel surface modification method for n-HA, so that it could improve the dispersion of n-HA and mechanical enhancement effect for PLGA, and it would have a great potential in the bone fracture internal fixation application in future.

\section{Materials and methods}

\subsection{Materials}

n-HA was prepared in our laboratory, whose average size of nanoparticles was about $100-120 \mathrm{~nm}$ in length and 20-40 nm in width. PLGA, whose copolymer composition (LA:GA) is 95:05 (mol:mol), $M_{\mathrm{w}}=$ $3.6 \cdot 10^{5}-3.9 \cdot 10^{5}$, was also prepared in our laboratory. Citric acid, AR, purchased from chemical reagent factory, Kelong, Chengdu. D, L-lactide (DLLA, Purac company) was recrystallized from ethyl acetate under argon atmosphere. Stannous octanoate was obtained from Sigma Company. All other reagents were of the analytical grade.

\subsection{Surface modification of $\mathrm{n}-\mathrm{HA}$ with the assist of citric acid}

$10 \mathrm{~g}$ citric acid in DMF was added into $10 \mathrm{~g}$ n-HA suspended in DMF with stirring. The reaction was maintained at $150^{\circ} \mathrm{C}$ for $11 \mathrm{~h}$ under nitrogen. The powder product was washed by ethanol and vacuum-dried, and $8 \mathrm{~g} \mathrm{n}$-HA product powder of surface-modified by citric acid was uniformly dispersed in dimethylbenzene by ultrasonic treatment. Then $8 \mathrm{~g}$ DLLA and $0.004 \mathrm{~g} \mathrm{Sn}(\mathrm{Oct})_{2}$ were added under nitrogen. The mixture was maintained at $140^{\circ} \mathrm{C}$ for $20 \mathrm{~h}$ under nitrogen. After it was washed with excessive dichloromethane and vacuum-dried, the obtained product powder was named as g1-n-HA. The n-HA of surface-grafted without citric acid was prepared in the same procedure as the control, which was named as g2-n-HA.

\subsection{Characteristics of surface-modified n-HA}

The IR analysis of unmodified n-HA and two surface-grafted n-HA powders were performed using Fourier transform infrared spectroscopy (Thermo Nicolet 670, USA) in KBr disks. 
The amount of grafted organic materials were determined by TGA (Perkin-Elmer 7 series thermal analysis system) at a heating rate of $10^{\circ} \mathrm{C} / \mathrm{min}$ from room temperature to $700^{\circ} \mathrm{C}$ under a high-purity nitrogen atmosphere.

$\mathrm{X}$-ray diffraction analysis were carried out by XRD powder diffractometer (Philips, X' Pert Pro, $\mathrm{Cu} \mathrm{K} \mathrm{K}_{\alpha}$ ). A scan axis of $2 \theta$ was used to obtain diffraction patterns of a scan range from 15 to $70^{\circ}$. The voltage used was $40 \mathrm{kV}$ and the current was $45 \mathrm{~mA}$.

TEM (JME-100CX, Seike Instruments) investigations were taken on a $200 \mathrm{kV}$ acceleration voltage. The specimens were prepared by dripping a drop of $0.1 \%$ suspension onto a TEM grid covered with carbon film and evaporated the solvent completely at room temperature.

The dispersion test was valued by the colloid stability according to the intuitive photographs taken by a conventional camera at different time. The powder was dispersed in dicholomathane $(1.0 \mathrm{~g} / \mathrm{L})$.

\subsection{Preparation of g-n-HA/PLGA composites}

The pure PLGA was dissolved in dichloromethane, and pre-calculated two surface-grafted n-HA uniformly dispersed in ethanol and dichloromethane were slowly added into PLGA solution by the addition of $3,8,15$ and $25 \mathrm{wt} \%$, respectively. Then the mixtures were stirred with ultrasonic treatment for more than $4 \mathrm{~h}$. The precipitates were washed with excessive ethanol and dried under vacuum at $40^{\circ} \mathrm{C}$ for $48 \mathrm{~h}$.

\subsection{Properties of g-n-HA/PLGA composites}

Fracture surfaces of PLGA after different addition contents of two surface-grafted were observed by SEM (S-520, Hitachi, Japan) after gold coating, which was used to investigate the dispersion of the g-n-HA nanoparticles in PLGA matrix and the interface microstructure.

The thermal properties of PLGA after different addition contents of two surface-grafted were measured by DSC (Q20, TA instruments, USA) at a heating rate of $10^{\circ} \mathrm{C} / \mathrm{min}$ from room temperature to $190^{\circ} \mathrm{C}$ with flowing nitrogen gas, holding at $190^{\circ} \mathrm{C}$ for $5 \mathrm{~min}$ to erase thermal history, then cooled to $40^{\circ} \mathrm{C}$ with $10^{\circ} \mathrm{C} / \mathrm{min}$ cooling rate, finally heated to $190^{\circ} \mathrm{C}$ with $10^{\circ} \mathrm{C} / \mathrm{min}$ rate again, and the second heat curve was analyzed. The crystallinity $\left(X_{\mathrm{c}}\right)$ was calculated according to Equation (1):
$X_{\mathrm{c}}=\frac{\Delta H_{\mathrm{m}}}{\omega \cdot \Delta H_{\mathrm{m}, 100 \%}}$

where $\Delta H_{\mathrm{m}}$ indicates the melting enthalpy, and $\omega$ is the weight fraction of PLGA in the composite. $\Delta H_{\mathrm{m}, 100 \%}$ is the theoretical enthalpy $(93.7 \mathrm{~J} / \mathrm{g})$ of completely crystalline poly(L-lactic acid) (PLLA) [21].

The mechanical enhancement effect for PLGA after different addition contents of two surface-grafted were measured by an electromechanical universal testing machine (CMT6000, Sans, China). According to GB/T1042-92, the three-point bending strength of rectangular bars of $4 \mathrm{~mm} \times 6 \mathrm{~mm} \times 60 \mathrm{~mm}$ were measured at a crosshead speed of $20 \mathrm{~mm} / \mathrm{min}$, and the tensile strength of specimens with dimensions of $50 \mathrm{~mm} \times 6 \mathrm{~mm} \times 0.5 \mathrm{~mm}$ were measured at a speed of $1 \mathrm{~mm} / \mathrm{min}$, at $22^{\circ} \mathrm{C}$ and a relative humidity of $60 \%$. The average value of five measurements was given.

\section{Results and discussion}

\subsection{Characterization of surface modification reactions for $\mathbf{n}-\mathbf{H A}$}

\subsubsection{IR analysis}

Figure 1 gives the FTIR spectra of unmodified n-HA and surface-modified n-HA. Some of the main absorption peaks of $\mathrm{PO}_{4}{ }^{3-}$, $-\mathrm{OH}$ groups and $\mathrm{H}_{2} \mathrm{O}$ were identified for n-HA (shown in Figure 1 curve a). In addition, the split peaks at 1420.0 and $1465.6 \mathrm{~cm}^{-1}$ belonging to $\mathrm{CO}_{3}{ }^{2-}$ asymmetric stretch peak are also observed, which was resulted from the minor $\mathrm{CO}_{3}{ }^{2-}$ inclusion during the n-HA synthesis. These results are in accordance with those of n-HA in literature [22]. After surface modification, besides these bands, for g2-n-HA exhibits bands of the carbonyl groups at $1737.5 \mathrm{~cm}^{-1}$, and some small peaks observed between 3000 and $2850 \mathrm{~cm}^{-1}$ are assigned

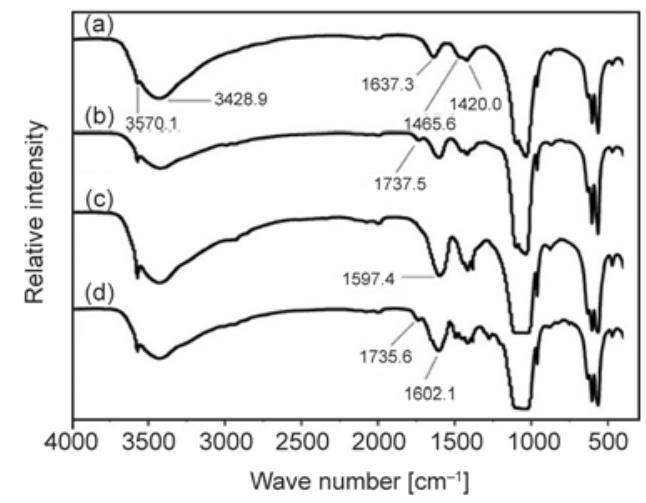

Figure 1. IR spectra of (a) unmodified n-HA, (b) g2-n-HA, (c) n-HA of citric acid modified, (d) g1-n-HA 
to $\mathrm{C}-\mathrm{H}$ stretching vibration of the $\mathrm{CH}_{3}-$ and $\mathrm{CH}-$ groups (shown in Figure 1 curve b), suggesting that ester bonds originated from the polymerization of DLLA on the n-HA surface existed. For the n-HA modified by citric acid (shown in Figure 1 curve c), small peaks of $\mathrm{C}-\mathrm{H}$ stretching vibration emerges and the peak of $\mathrm{COO}^{-}$at $1597.4 \mathrm{~cm}^{-1}$ is observed, implying that calcium carboxylate on the surface of n-HA was formed. While g1-n-HA exhibits a new absorption peak at $1602.1 \mathrm{~cm}^{-1}$ belonging to carbonyl groups of PLA, besides for the carbonyl groups at $1735.6 \mathrm{~cm}^{-1}$ (shown in Figure 1 curve d). According to the above analysis, we concluded that citric acid and PLA were both successfully grafted onto the surface of n-HA.

\subsubsection{TGA}

The amount of grafted organic substances is determined by TGA, as shown in Figure 2. According to the TGA curves, the weight losses of unmodified n-HA, n-HA of citric acid modified and two g-n-HA are $4.703,8.06 \%, 12.86$ and $6.303 \%$, respectively. The surface-grafted amount onto n-HA is calculated as follows by the equation, the grafting amounts $[\%]=W_{1}[\%]-W_{0}[\%]$, where $W_{1}$ is the weight loss of surface-grafted n-HA and $W_{0}$ is the weight loss of unmodified n-HA. Therefore, the total grafting amounts onto n-HA of citric acid, g1-n-HA and g2-n-HA are 3.357, 8.157 and $1.600 \%$, respectively, and the grafting amounts of PLA onto g1-n-HA and g2-n-HA are $4.800(8.157 \%-3.357 \%)$ and $1.600 \%$, respectively. Obviously, it is further proved that $n-H A$ surface was successful grafted quantitatively. Moreover, the grafting amount of g1-n-HA is larger than that of g2-n-HA, which shows that citric acid played an important role in increasing grafting amounts of PLA onto n-HA, and the reason may be attributed to the higher reactivity of the

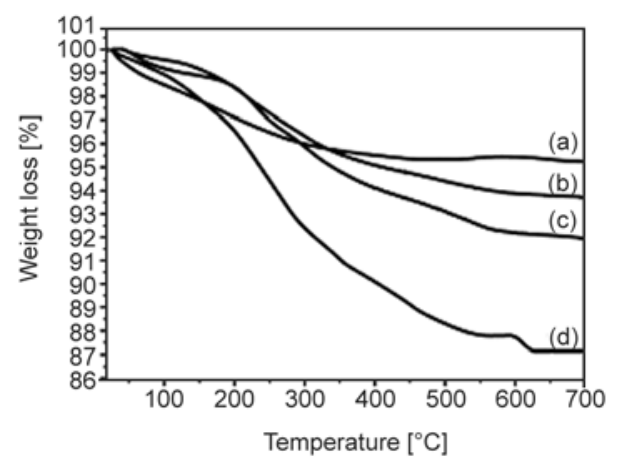

Figure 2. TGA curves of (a) unmodified n-HA, (b) g2-n-HA, (c) n-HA of citric acid modified, (d) g1-n-HA hydroxyl groups of citric acid and the better dispersion of n-HA due to the steric hindrance of citric acid during the processing of grafting with $\mathrm{D}$, L-lactide, comparing with n-HA directly grafted with $\mathrm{D}$, L-lactide without citric acid. The higher grafting amounts onto n-HA would be benefit to improve the dispersion of n-HA and interface adhesion with PLGA matrix when it was added into PLGA [23].

\subsubsection{XRD}

Figure 3 gives the XRD patterns of unmodified n-HA and two g-n-HA. The unmodified n-HA exhibits characteristic peaks at $2 \theta$ regions of $26,32-34,40$, and $46-54^{\circ}$, which is consistent with the crystalline nature of n-HA in literature [24]. Comparing the XRD patterns of g-n-HA with unmodified n-HA, it can be found that the characteristic diffractions of two g-n-HA nanocrystals are nearly the same as those of unmodified n-HA, which shows that there are no other new apatite crystalline phases, namely, the surface modifications didn't significantly affect the crystalline phases of n-HA. However, it is easy to find that the intensity of characteristic crystalline peaks of $2 \theta$ at $26^{\circ}$ becomes a little weaker after being modified, and the crystalline peaks of g1-n-HA is weaker than that of g2-n-HA, whose crystallinity of unmodified n-HA, g1-n-HA and g2-n-HA calculated with XRD analysis software Jade are 80.81 , 72.00 and $80.53 \%$, respectively. The difference of crystallinity is attributed to the covering of the organic substances grafted on n-HA, and the larger the grafting amount is, the lower the crystallinity of $\mathrm{n}-\mathrm{HA}$ is, which is in agreement with the results of TGA.

Based on these, the surface-grafting reaction with citric acid for n-HA are assumed as shown in Figure 4. First, $\mathrm{Ca}$ on the surface of n-HA reacted with car-

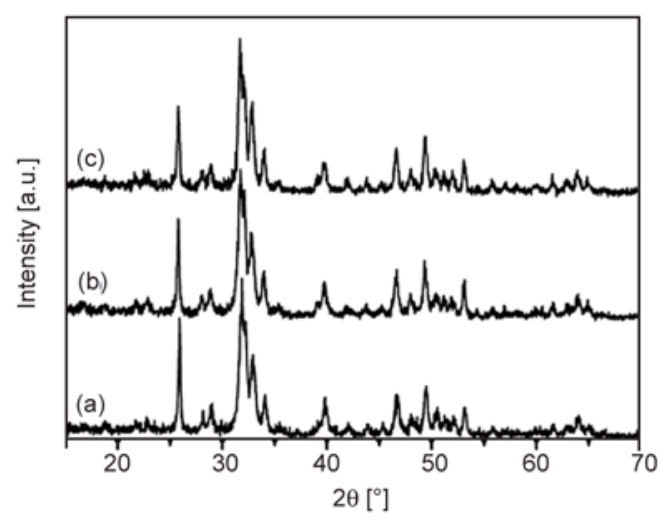

Figure 3. XRD patterns of (a) unmodified n-HA, (b) g2-n-HA, (c) g1-n-HA 


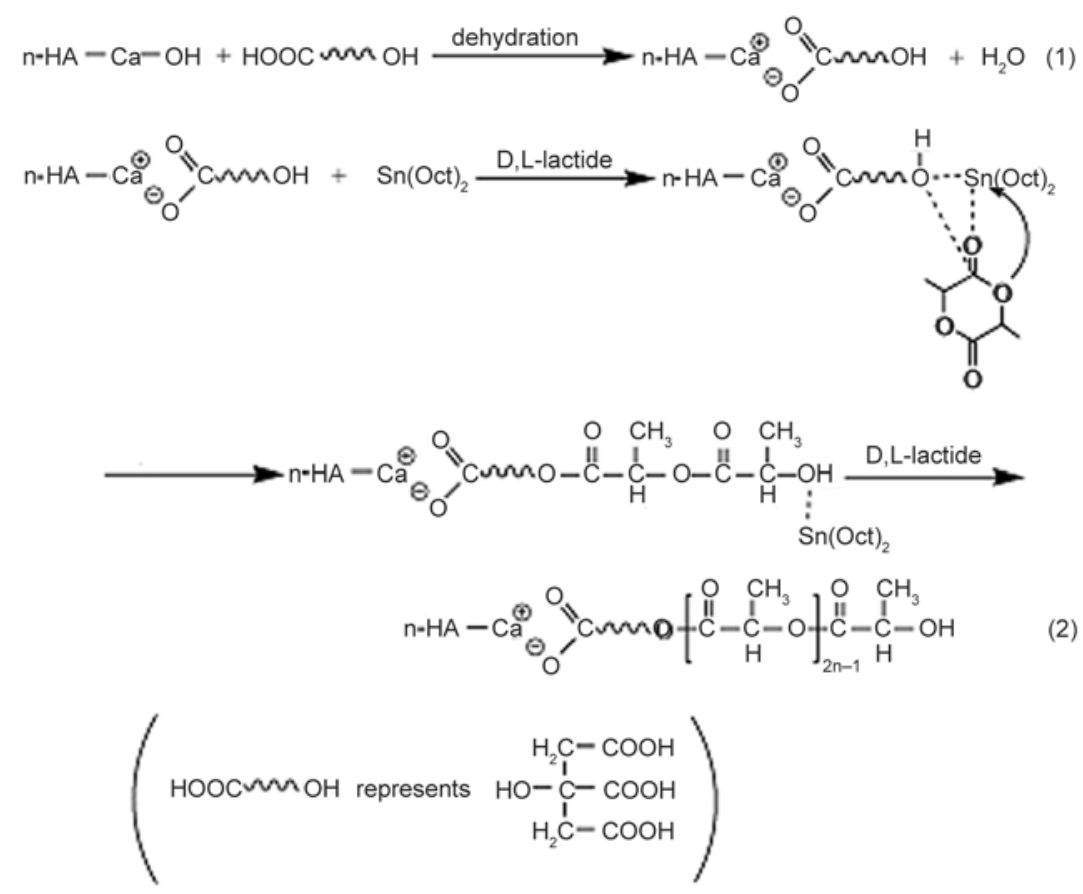

Figure 4. Illustration of the surface grafting with citric acid for n-HA

boxylate of citric acid to form a Ca Carboxylate bond (Step (1)). Then the propagation reaction in the presence of $\mathrm{Sn}(\mathrm{Oct})_{2}$ included in succession the coordination of both the lactide and the hydroxyl group of calcium citric acid on the surface of HA modified by citric acid with $\mathrm{Sn}(\mathrm{Oct})_{2}$ followed by cleavage of the acyl-oxygen bond of the D, L-lactide by the Sn-coordinate insertion mechanism and thereby activated hydroxyl group as indicated in (Step (2)) [20].

\subsubsection{TEM}

TEM photographs of unmodified n-HA and two g-n-HA are shown in Figure 5. It can be seen that they are all needles, indicating that the morphology of g-n-HA nanoparticles did not change after being surface-modified. However, unmodified n-HA nanoparticles exhibit aggregation phenomenon in dichloromethane (seen in Figure 5a), while the dispersion of two g-n-HA are obviously improved after being surface-modified, as shown in Figure $5 \mathrm{~b}$ and

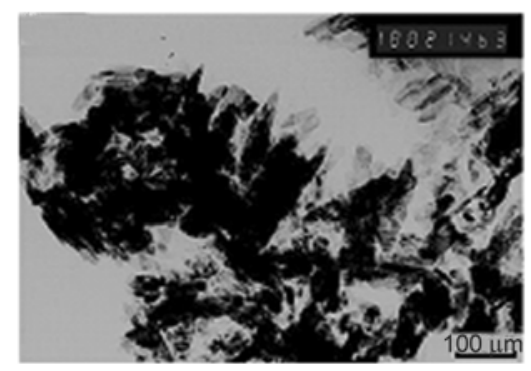

a)

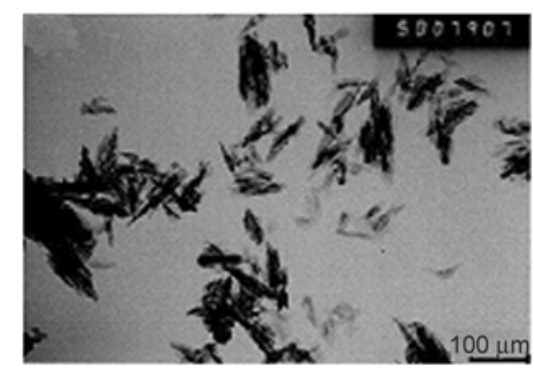

b) 5c. Especially, g1-n-HA has better dispersibility than g2-n-HA, which may be resulted from its higher grafting amount.

\subsubsection{Dispersion test}

To further investigate the dispersion effect of surface modification for n-HA, the three different samples were dispersed in dichloromethane, and the intuitive photographs taken by a conventional camera are given in Figure 6. As expected, unmodified n-HA particles precipitated in hydrophobic organic solution immediately after ultrasonication. However, after n-HA particles was coated by PLA chains, the surface wettability was greatly improved, so that the two g-n-HA could maintain a stable dispersion in suspension for a longer time. Especially, g1-n-HA exhibits more excellent colloid stability, which can remain stable for one day, while a little precipitate appears in the suspension of g2-n-HA. Obviously, the dispersion of g-n-HA is closely related

Figure 5. TEM photographs of (a) unmodified n-HA, (b) g2-n-HA, (c) g1-n-HA

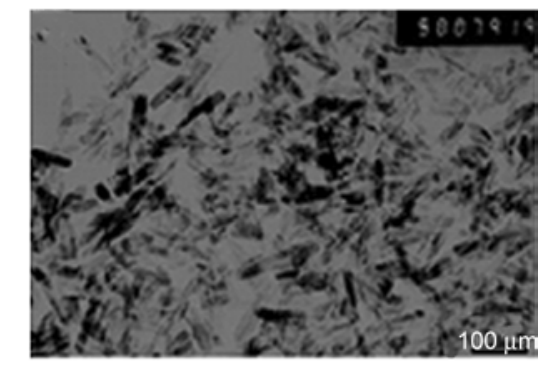

c) 

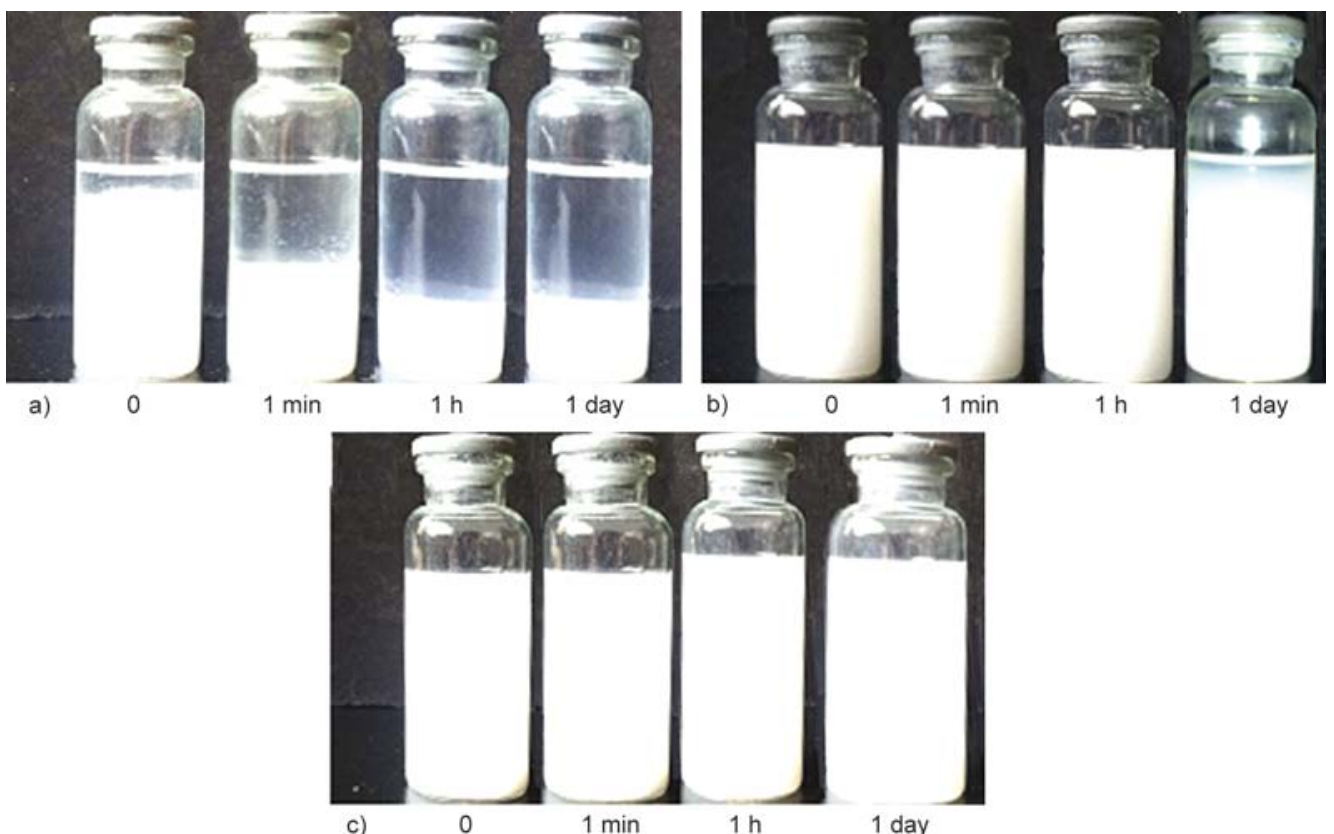

1 day

b)

day

c)

0

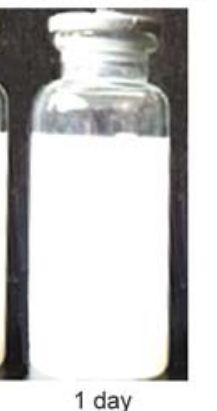

Figure 6. Photographs of (a) unmodified n-HA, (b) g2-n-HA, (c) g1-n-HA dispersed in dichloromethane for different time

to the grafting amount on the surface of n-HA. This result shows that the novel method of surfacegrafted with the assistance of citric acid for n-HA could change the surface wettability and prevent the aggregation of n-HA particles is more efficient than the method of the directly surface-grafted LA without citric acid for n-HA, and the dispersibility is better than some literature [25], which may be more useful to enhance mechanical properties for PLGA.

\subsection{Properties of g-n-HA/PLGA composites}

\subsubsection{SEM observation}

To investigate the dispersion of g-n-HA particles in PLGA matrix and the interfacial adhesion, the SEM

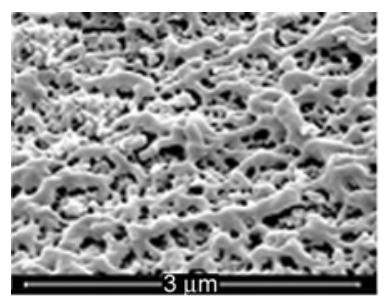

a)

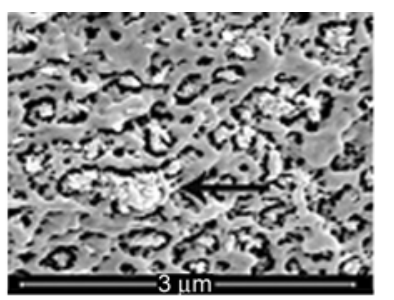

b)

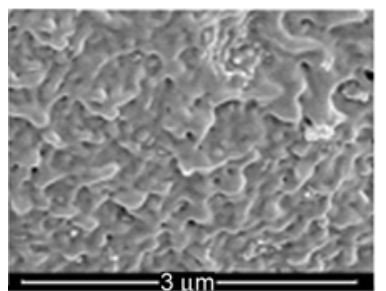

A)

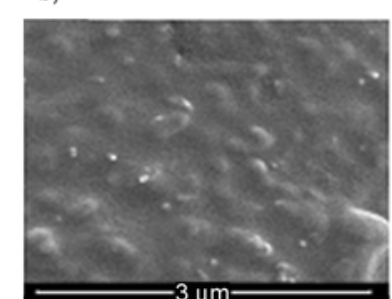

B)

analysis has been performed. The SEM micrographs are given in Figure 7. For g2-n-HA/PLGA composite, there are many cavities between g2-n-HA particles and PLGA matrix, and many agglomerated g2-n-HA particles emerge when the g2-n-HA content is up to $8 \mathrm{wt} \%$, indicating that there was poor dispersion and interfacial adhesion. However, for the g1-n-HA/PLGA composite, there are few cavities and agglomerated particles until the g1-n-HA content increased to $25 \mathrm{wt} \%$. Obviously, it can be concluded that the g1-n-HA dispersed much more uniformly in PLGA matrix than g2-n-HA, so surface-grafted with the assistance of citric acid for n-HA is a novel surface-grafting method to improve

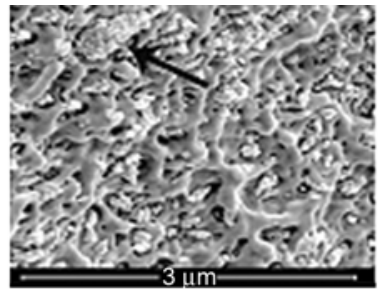

c)

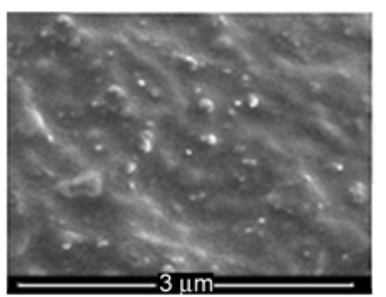

C)

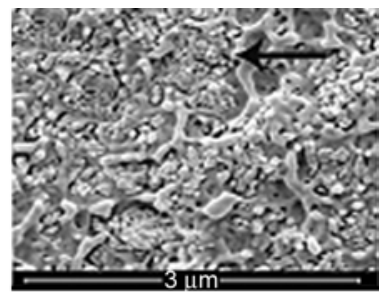

d)

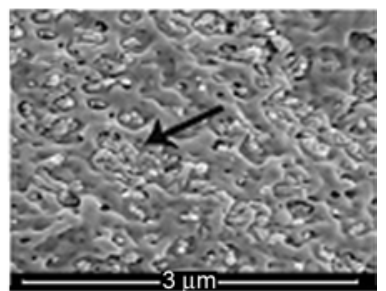

D)

Figure 7. SEM micrographs of different g-n-HA addition content of g2-n-HA at (a) $3 \mathrm{wt} \%$, (b) $8 \mathrm{wt} \%$, (c) $15 \mathrm{wt} \%$, (d) $25 \mathrm{wt} \%$ and g1-n-HA (A) $3 \mathrm{wt} \%$, (B) $8 \mathrm{wt} \%$, (C) $15 \mathrm{wt} \%$, (D) $25 \mathrm{wt} \%$. The arrows show the agglomerate g-n-HA particles in PLGA matrix 
the dispersion of nanoparticles in PLGA matrix and the interfacial adhesion, which has a close relationship with the mechanical enhancement effect for PLGA [26].

\subsubsection{Thermal properties}

To further investigate the thermal properties, the DSC curves of PLGA after the different addition of two g-n-HA are shown in Figure 8, and the thermal properties data calculated from DSC curves are listed in Table 1. It can be found that the melting temperature $\left(T_{\mathrm{m}}\right)$ seems to have no relationship with the loading of g-n-HA particles, while the crystallinity of PLGA matrix increased with the increasing g-n-HA content. What is more, the crystallinity of PLGA after the addition of g1-n-HA is higher than that of corresponding addition of g2-n-HA, which indicates the g1-n-HA acts as a more effective heterogeneous nucleating agent than g2-n-HA. The reason is that the better dispersion of $\mathrm{g} 1-\mathrm{n}-\mathrm{HA}$ in PLGA matrix, the more conducive it is to absorb PLGA matrix onto the surface of g1-n-HA particles,

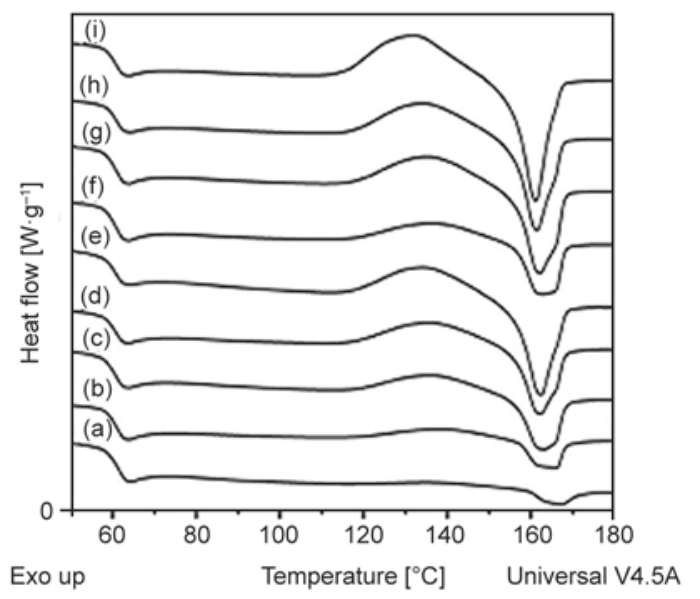

Figure 8. DSC thermograms of samples of (a) PLGA, (b) $3 \mathrm{wt} \%$, (c) $8 \mathrm{wt} \%$, (d) $5 \mathrm{wt} \%$, (e) $25 \mathrm{wt} \%$ of g2-n-HA, (f) $3 \mathrm{wt} \%$, (g) $8 \mathrm{wt} \%$, (h) $15 \mathrm{wt} \%$, (i) $25 \mathrm{wt} \%$ of g1-n-HA so that the nucleation and crystallinity are further promoted, which may be another factor to enhance the mechanical property for PLGA [27].

\subsubsection{Mechanical properties}

The initial mechanical property is usually a critical technical index for bone fracture internal fixation materials. The enhancement dependency of bending strength and tensile strength for PLGA after different addition content of two g-n-HA are shown in Figure 9. Obviously, it can be seen that the maximum enhancement of bending strength and tensile strength is at $3 \mathrm{wt} \%$ surface-grafted, and it leads to a reduction in the bending strength and tensile strength with the further increasing g-n-HA content. Moreover, g1-n-HA has more improvement in mechanical properties than g2-n-HA, whose bending strength and tensile strength were both over $20 \%$ higher than those of pure PLGA when $3 \mathrm{wt} \%$ g1-n-HA was added. Especially, even $15 \mathrm{wt} \%$ g1-n-HA was added, whose bending and tensile strength are still 8 and $6 \%$ higher than those of pure PLGA, respectively. However, in our previous study, the maximum addition content of n-HA into PLGA was only $10 \mathrm{wt} \%$, and the addition of $15 \mathrm{wt} \% \mathrm{n}-\mathrm{HA}$ decreased markedly its bending strength $[18,19]$. Obviously, the mechanical enhancement for PLGA is by far the most excellent as comparing to some related literatures [28]. The main reason may also be ascribed to the higher grafting amount for g1-n-HA, which might improve the dispersion of g1-n-HA nanoparticles in PLGA matrix and the interfacial adhesion as well as the increased crystallinity for PLGA.

\section{Conclusions}

In this study, a new method of surface-grafting D, L-lactide (DLLA) with the assistance of citric acid

Table 1. Thermal properties of PLGA after different addition content of two g-n-HA determined by DSC

\begin{tabular}{|c|c|c|c|c|}
\hline Sample & $\begin{array}{c}\text { Addition g-n-HA content } \\
{[w t \%]}\end{array}$ & $\begin{array}{c}\mathbf{T}_{\mathrm{m}} \\
{\left[{ }^{\circ} \mathbf{C}\right]}\end{array}$ & $\begin{array}{c}\Delta \mathbf{H}_{\mathrm{m}} \\
{\left[\mathrm{J} \cdot \mathrm{g}^{-1}\right]}\end{array}$ & $\begin{array}{c}\mathbf{X}_{\mathrm{c}} \\
{[\%]}\end{array}$ \\
\hline PLGA & 0 & 166.78 & 2.423 & 2.586 \\
\hline \multirow{4}{*}{ g2-n-HA/PLGA } & 3 & 164.24 & 5.221 & 5.744 \\
\hline & 8 & 161.98 & 10.45 & 12.12 \\
\hline & 15 & 161.68 & 13.99 & 17.57 \\
\hline & 25 & 161.93 & 18.14 & 25.81 \\
\hline \multirow{4}{*}{ g1-n-HA/PLGA } & 3 & 161.86 & 9.623 & 10.59 \\
\hline & 8 & 161.71 & 16.05 & 18.62 \\
\hline & 15 & 161.13 & 18.56 & 23.30 \\
\hline & 25 & 160.92 & 22.96 & 32.67 \\
\hline
\end{tabular}



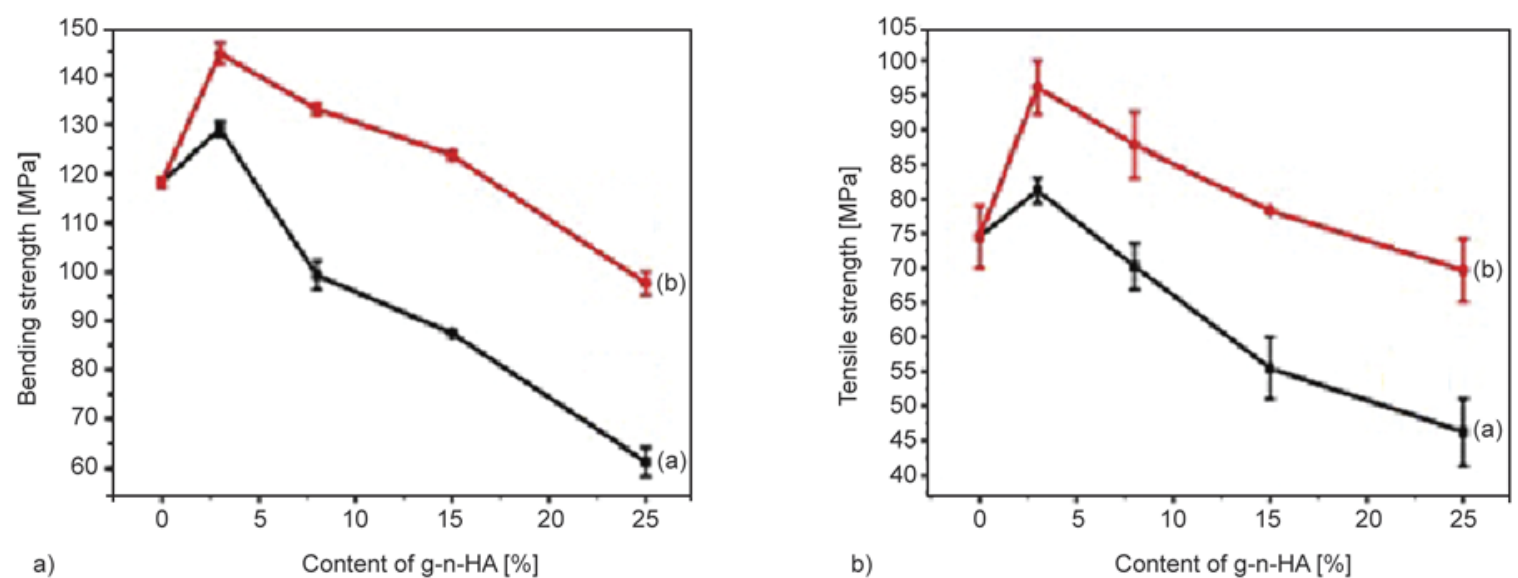

Figure 9. Mechanical properties of different g-n-HA content (a) bending strength, (b) tensile strength (red curve is g1-n-HA, and black curve is g2-n-HA)

for n-HA was designed, comparing with the surface-grafting without citric acid, and the two surface-grafted n-HA were respectively introduced into PLGA matrix with different contents. The results showed that the two surface-grafting methods for n-HA were both successful. However, citric acid played a critical role in surface-grafting, which had much higher grafting amount and better dispersion than surface-grafting without citric acid, so that the g-n-HA/PLGA composites exhibited more excellent mechanical properties owing to the better dispersion and interfacial adhesion throughout PLGA matrix, and the higher crystallinity for PLGA, whose bending strength and tensile strength were both over $20 \%$ higher than those of pure PLGA when $3 \mathrm{wt} \% \mathrm{n}-\mathrm{HA}$ was added. Especially, even $15 \mathrm{wt} \%$ n-HA was added, whose bending and tensile strength were still 8 and $6 \%$ higher than those of PLGA, respectively. The above results suggested that the new surface-grafting with the aid of citric acid for n-HA was an ideal novel surface modification method, which could greatly improve the dispersion and achieve a better mechanical strength for g-n-HA/PLGA composite with higher n-HA content, suggesting that it has a great potential in the bone fracture internal fixation application in future. For the degradability and biological properties of the g-n-HA/PLGA composite, the work is in progress, and the results would be reported in another paper in the future.

\section{Acknowledgements}

This work was supported by the Talent Training Project of West Light Foundation of Chinese Academy of Science, and National Natural Science Foundation (31000440).

\section{References}

[1] Raikin S. M., Ching A. C.: Bioabsorbable fixation in foot and ankle. Foot and Ankle Clinics, 10, 667-684 (2005). DOI: $10.1016 / \mathrm{j} . \mathrm{fcl} \cdot 2005.06 .008$

[2] Saito E., Liao E. E., Hu W-W., Krebsbach P. H., Hollister S. J.: Effects of designed PLLA and 50:50 PLGA scaffold architectures on bone formation in vivo. Journal of Tissue Engineering and Regenerative Medicine, 7, 99-111 (2013).

DOI: $10.1002 /$ term.497

[3] Tiainen J., Soini Y., Törmälä P., Waris T., Ashammakhi N.: Self-reinforced polylactide/polyglycolide $80 / 20$ screws take more than $1 \frac{1 / 2}{2}$ years to resorb in rabbit cranial bone. Journal of Biomedical Materials Research Part B: Applied Biomaterials, 70, 49-55 (2004). DOI: $10.1002 / \mathrm{jbm} \cdot$ b. 30013

[4] Swetha M., Sahithi K., Moorthi A., Srinivasan N., Ramasamy K., Selvamurugan N.: Biocomposites containing natural polymers and hydroxyapatite for bone tissue engineering. International Journal of Biological Macromolecules, 47, 1-4 (2012). DOI: $10.1016 /$ j.ijbiomac.2010.03.015

[5] Sun F., Zhou H., Lee J.: Various preparation methods of highly porous hydroxyapatite/polymer nanoscale biocomposites for bone regeneration. Acta Biomaterialia, 7, 3813-3828 (2001). DOI: $10.1016 /$ j.actbio.2011.07.002

[6] Wang D. X., He Y., Bi L., Qu Z. H., Zou J. W., Pan Z., Fan J. J., Chen L., Dong X., Liu X. N., Pei G. X., Ding J. D.: Enhancing the bioactivity of poly(lactic-co-glycolic acid) scaffold with a nano-hydroxyapatite coating for the treatment of segmental bone defect in a rabbit model. International Journal of Nanomedicine, 8 , 1855-1865 (2013).

DOI: $10.2147 /$ IJN.S43706 
[7] Kim S-S., Park M. S., Jeon O., Choi C. Y., Kim B-S.: Poly(lactide-co-glycolide)/hydroxyapatite composite scaffolds for bone tissue engineering. Biomaterials, 27, 1399-1409 (2006).

DOI: 10.1016/j.biomaterials.2005.08.016

[8] Petricca S. E., Marra K. G., Kumta P. N.: Chemical synthesis of poly(lactic-co-glycolic acid)/hydroxyapatite composites for orthopaedic applications. Acta Biomaterialia, 2, 277-286 (2006).

DOI: $10.1016 /$ j.actbio.2005.12.004

[9] Šupová M.: Problem of hydroxyapatite dispersion in polymer matrices: A review. Journal of Materials Science: Materials in Medicine, 20, 1201-1213 (2009).

DOI: $10.1007 / \mathrm{s} 10856-009-3696-2$

[10] Borum L., Wilson O. C.: Surface modification of hydroxyapatite. Part II. Silica. Biomaterials, 24, 36813688 (2003).

DOI: $10.1016 / \mathrm{S} 0142-9612(03) 00240-0$

[11] Liu Q., de Wijn J. R., Bakker D., van Toledo M., van Blitterswijk C. A.: Polyacids as bonding agents in hydroxyapatite polyester-ether (PolyactiveTM 30/70) composites. Journal of Materials Science: Materials in Medicine, 9, 23-30 (1998).

DOI: $10.1023 / \mathrm{A}: 1008826410395$

[12] Dong G-C., Sun J-S., Yao C-H., Jiang G. J., Huang CW., Lin F-H.: A study on grafting and characterization of HMDI-modified calcium hydrogenphosphate. Biomaterials, 22, 3179-3189 (2001).

DOI: 10.1016/S0142-9612(01)00070-9

[13] Wang Y., Zhang X., Yan J., Xiao Y., Lang M.: Surface modification of hydroxyapatite with poly(methyl methacrylate) via surface-initiated ATRP. Applied Surface Science, 257, 6233-6238 (2011).

DOI: $10.1016 /$ j.apsusc.2011.02.045

[14] Hong Z., Zhang P., Liu A., Chen L., Chen X., Jing X.: Composites of poly(lactide-co-glycolide) and the surface modified carbonated hydroxyapatite nanoparticles. Journal of Biomedical Materials Research: Part A, 81, 515-522 (2007).

DOI: $10.1002 / j b m . a .31038$

[15] Cui Y., Liu Y., Cui Y., Jing X., Zhang P., Chen X.: The nanocomposite scaffold of poly(lactide-co-glycolide) and hydroxyapatite surface-grafted with L-lactic acid oligomer for bone repair. Acta Biomaterialia, 5, 2680 2692 (2009).

DOI: $10.1016 /$ j.actbio.2009.03.024

[16] Lock J., Nguyen T. Y., Liu H. N.: Nanophase hydroxyapatite and poly(lactide-co-glycolide) composites promote human mesenchymal stem cell adhesion and osteogenic differentiation in vitro. Journal of Materials Science: Materials in Medicine, 23, 2543-2552 (2012). DOI: $10.1007 / \mathrm{s} 10856-012-4709-0$

[17] Jiang L., Xiong C., Chen D., Jiang L., Pang X.: Effect of n-HA with different surface-modified on the properties of n-HA/PLGA composite. Applied Surface Science, 259, 72-78 (2012).

DOI: $10.1016 /$ j.apsusc.2012.06.091
[18] Jiang L., Xiong C., Jiang L., Chen D., Li Q.: Effect of n-HA content on the isothermal crystallization, morphology and mechanical property of n-HA/PLGA composites. Materials Research Bulletin, 48, 1233-1238 (2013).

DOI: 10.1016/j.materresbull.2012.11.113

[19] Jian L-X., Jiang L-Y., Ma C., Han C-T., Xu L-J., Xiong C-D.: Preparation and characterization of nanohydroxyapatite/plga composites with novel surfacemodified nano-hydroxyapatite. Jounal of Inorganic Materials, 28, 751-756 (2013).

DOI: 10.3724/SP.J.1077.2013.12502

[20] Qiu X., Hong Z., Hu J., Chen L., Chen X., Jing X.: Hydroxyapatite surface modified by L-lactic acid and its subsequent grafting polymerization of L-lactide. Biomacromolecules, 6, 1193-1199 (2005).

DOI: $10.1021 / \mathrm{bm} 0495021$

[21] Wu H., Pang D., Ma C., Li Q., Xiong C.: Composites of hydroxyapatite whiskers/poly(L-lactide-co-glycolide) with high tensile plasticity. Journal of Macromolecular Science Part B: Physics, 51, 1242-1255 (2011). DOI: 10.1080/00222348.2011.627824

[22] Lee J. H., Shofner M. L.: Copolymer-mediated synthesis of hydroxyapatite nanoparticles in an organic solvent. Langmuir, 29, 10940-10944 (2013).

DOI: $10.1021 / 1 \mathrm{a} 402434 \mathrm{v}$

[23] Hong Z., Qiu X., Sun J., Deng M., Chen X., Jing X.: Grafting polymerization of L-lactide on the surface of hydroxyapatite nano-crystals. Polymer, 45, 66996706 (2004).

DOI: $10.1016 /$ j.polymer.2004.07.036

[24] Boanini E., Fini M., Gazzano M., Bigi A.: Hydroxyapatite nanocrystals modified with acidic amino acids. European Journal of Inorganic Chemistry, 23, 48214826 (2006).

DOI: $10.1002 /$ ejic. 200600423

[25] Wei J., Liu A., Chen L., Zhang P., Chen X., Jing X.: The surface modification of hydroxyapatite nanoparticles by the ring opening polymerization of $\gamma$-benzyl-Lglutamate $N$-carboxyanhydride. Macromolecular Bioscience, 9, 631-638 (2009).

DOI: $10.1002 / \mathrm{mabi} .200800324$

[26] Zhang Y., Meng B., Chen L., Tao J., Wu Z.: Properties and structures of polylactide filled with poly(E-caprolactone)-coated calcium carbonate. Journal of Applied Polymer Science, 125, 952-958 (2012).

DOI: $10.1002 / a p p .36280$

[27] Luo Y-B., Li W-D., Wang X-L., Xu D-Y., Wang Y-Z.: Preparation and properties of nanocomposites based on poly(lactic acid) and functionalized $\mathrm{TiO}_{2}$. Acta Materialia, 57, 3182-3191 (2009).

DOI: $10.1016 /$ j.actamat.2009.03.022

[28] Hong Z., Zhang P., He C., Qiu X., Liu A., Chen L., Chen X., Jing X.: Nano-composite of poly(L-lactide) and surface grafted hydroxyapatite: Mechanical properties and biocompatibility. Biomaterials, 26, 62966304 (2005).

DOI: $10.1016 /$ j.biomaterials.2005.04.018 